\title{
Criminologie
}

\section{La prédiction de la délinquance}

\section{Rolf Loeber et Magda Stouthamer-Loeber}

Volume 19, numéro 2, 1986

La prédiction de la carrière criminelle

URI : https://id.erudit.org/iderudit/017240ar

DOI : https://doi.org/10.7202/017240ar

Aller au sommaire du numéro

Éditeur(s)

Les Presses de l'Université de Montréal

ISSN

0316-0041 (imprimé)

1492-1367 (numérique)

Découvrir la revue

Citer cet article

Loeber, R. \& Stouthamer-Loeber, M. (1986). La prédiction de la délinquance. Criminologie, 19(2), 49-77. https://doi.org/10.7202/017240ar

\section{Résumé de l'article}

The paper presents the results of a meta-analysis to analyze which predictors of delinquency in males and females are consistently evident from longitudinal studies, and how the predictors compare in their predictive efficiency. The results show that a variety of conduct problems but also conditions within the family are predictive of later delinquency in general, recidivism, or serious offenses. As a rule, composite indicators of risk factors outperformed single indicators. Data is presented on the early identification of chronic offenders. The results are critically examined in terms of error rates, and in terms of future directions for research 
The paper presents the results of a meta-analysis to analyze which predictors of delinquency in males and females are consistently evident from longitudinal studies, and how the predictors compare in their predictive efficiency. The results show that a variety of conduct problems but also conditions within the family are predictive of later delinquency in general, recidivism, or serious offenses. As a rule, composite indicators of risk factors outperformed single indicators. Data is presented on the early identification of chronic offenders. The results are critically examined in terms of error rates, and in terms of future directions for research.

\section{INTRODUCTION}

Dans son ouvrage novateur sur la prédiction du comportement violent, Monaham (1981) a écrit que le genre humain n'aurait probablement pas survécu aussi bien si les hommes n'avaient tenté de prédire l'impact de la nature sur eux-mêmes. Les humains ont appris que, puisque la plupart des lions mordaient et que les éboulis écrasaient, il valait mieux éviter les uns et les autres. Et de la même manière ils ont essayé de prédire la conduite d'autrui, particulièrement lorsque des individus étaient susceptibles d'en faire des victimes, en les blessant ou en les privant de leur propriété.

On peut utiliser de bien des façons l'information prédictive. Premièrement, les fonctionnaires de la cour, les intervenants sociaux et les parents s'en servent pour évaluer quels jeunes sont les plus prédisposés à commettre des délits ou à devenir des délinquants persistants et ont, de la sorte, besoin d'une intervention plus poussée. Ce genre d'information peut servir à une deuxième fin, qui consiste à aider les auteurs de politiques criminelles et les chercheurs à préciser quels groupes de jeunes

* Extrait d'un livre en préparation, intitulé Handbook of Juvenile Delinquency. H. Quay (édit.), John Wiley \& Sons Inc., traduit de l'anglais par Suzanne LaflammeCusson.

** et*** Les auteurs sont professeurs adjoints au Western Psychiatric Institute and Clinic School of Medicine, University of Pittsburgh. 
risquent le plus de faire carrière dans la délinquance et, dès lors, doivent recevoir davantage de services spéciaux. Enfin, les données prédictives remplissent un troisième objectif en permettant de tester la pertinence des théories sur le développement de la délinquance.

\section{LA PRÉDICTION DE LA CONDUITE INDIVIDUELLE}

On a systématiquement utilisé les données prédictives de délinquance en matière de libération conditionnelle, de probation et de cautionnement (Simon, 1971; Gottfredson et Gottfredson, 1984). Mais leur utilisation a été moins concluante lorsqu'il s'est agi de prédire la dangerosité ou la violence individuelle (Monahan, 1978; 1981). Récemment, on a suggéré d'utiliser les données prédictives en vue de la neutralisation sélective des délinquants. Il s'agit d'identifier parmi les auteurs de délits, les plus prolifiques et de leur imposer de plus longues sentences, dans le but d'abaisser le niveau général de la criminalité (Greenwood et Abrahamse, 1982). Un autre champ, moins exploré, est l'identification, par les parents, enseignants et autres intervenants, des jeunes qui risquent de devenir délinquants (e.g. Loeber et al., 1984; Spivack, 1983). L'idée commune sousjacente à ces démarches est qu'une fois identifiés les sujets à haut risque, on interviendrait pour les écarter de l'avenir qu'on redoute pour eux.

\section{L'IDENTIFICATION DES GROUPES À RISQUES}

Le besoin d'identifier les groupes à haut risque de délinquance se fait de plus en plus sentir chez les définisseurs de politique criminelle et chez les chercheurs. Habituellement, ces professionnels visent l'identification précoce des groupes à haut risque en termes de violence ou de chronicité de l'agir délinquant en vue, soit de l'étude nosologique poussée de ces groupes, soit de les soumettre à un traitement spécial pour contrer la tendance prévue. L'identification du risque est ici définie comme la capacité d'identifier des groupes dont le sujet moyen, s'il ne présente pas de problèmes ou en présente seulement quelques aspects actuellement, présentera plus tard, selon toute probabilité, le problème dans son entier (Bell et Pearl, 1982). On tient de plus en plus à identifier les risques lors de l'élaboration des politiques, que ce soit en santé mentale ou en 
matière d'administration de la justice criminelle, afin de concentrer les ressources financières et le développement de programmes là où les risques sont élevés. Les tenants d'une soi-disant imprévisibilité inhérente au comportement délinquant n'ont peut-être pas bien saisi que la cohérence à travers le temps ne signifie pas nécessairement des comportements identiques. S'il saute aux yeux que le vol en bas âge peut être relié au vol subséquent, il est moins évident que l'école buissonnière dans l'enfance puisse l'être aux actes délinquants ultérieurs. Dans un contexte différent, Kagan (1971) a parlé de continuité hétérotypique c'est-à-dire que des comportements présentant des phénotypes différents sont liés, à travers le temps, en un seul cadre conceptuel théorique. Dans la même ligne de pensée Emmerich (1964) a fait une distinction entre stabilité et continuité. La stabilité est le degré auquel les individus maintiennent leur position relative sur une caractéristique à travers le temps (ici on réfère aux problèmes de conduite ou à la délinquance). La continuité pour sa part, réfère au maintien de la qualité ou de la signification du comportement à travers le temps. Selon cette conceptualisation, la continuité peut prendre la forme ou d'une diversification, ou d'une aggravation, ou d'une atténuation des problèmes de conduite ou de l'agir délinquant. Croissance et décroissance sont des points à retenir pour les théories évolutionnistes de la prédiction de la délinquance. Comme Farrington (1976) l'a exprimé succinctement: "la fonction virtuellement la plus utile des études de prédiction criminologiques est de mettre à l'épreuve la validité des théories rivales sur les causes du crime et de la délinquance (p. 3). En ce sens, les théories de la délinquance doivent spécifier quels facteurs font que les uns deviennent délinquants avec le temps, que d'autres continuent et qu'enfin un dernier groupe renonce à ce genre d'activités et, de la sorte, rendre compte des différences individuelles de niveau de criminalité (Blumstein et al., 1985).

La possibilité de prédire la délinquance suscite des réactions divergentes. Certains prônent l'identification précoce des enfants à risque et l'intervention préventive (e.g. Chaiken et Chaiken, 1984). D'autres s'y opposent, craignant que les prophéties créatrices transforment des enfants innocents en criminels (Lemert, 1951; Tannenbaum, 1938). Un troisième groupe, plus académique, $s$ 'est fait défenseur de la prédiction, au moins sur papier, en vue de vérifier les théories sur la délinquance. 
Le but de cet article est d'étoffer les éléments prédicteurs de la délinquance juvénile et adulte avec les informations rassemblées au cours de l'enfance et de l'adolescence. Dans ce sens, cela permet de déborder nos travaux antérieurs sur ces prédicteurs (Loeber et Dishion, 1983; Loeber et StouthameLoeber, sous presse). La plupart des études existantes s'appuient sur l'évaluation du comportement de l'enfant à la maison, à l'école ou à la clinique. Les évaluations des enfants en prison sont plus rares et nous les excluons de notre propos. Nous retiendrons comme prédicteurs les comportements manifestes évidents et non les évaluations de personnalité ou psychophysiologiques faites à partir de questionnaires.

On distinguera entre les prédicteurs comportementaux des variables relatives à des patterns de comportement chez les jeunes, et les prédicteurs circonstanciels, des variables du milieu (Toby, 1961). Pour mesurer la délinquance, on utilisera aussi bien les dossiers de police et de cour que l'agir délinquant révélé par questionnaire. On fera des distinctions entre agir délinquant en général, délinquance grave et récidive, en s'attardant tout particulièrement à la prédiction de la délinquance persistante définie par au moins quatre arrestations ou quatre déclarations de culpabilité.

\section{LES PRÉDICTEURS DE LA DÉLINQUANCE}

Pour comparer plusieurs prédicteurs à travers diverses recherches, nous allons utiliser un indice appelé Amélioration relative sur le hasard (ARH). Il s'agit d'une mesure d'association, entre deux variables dichotomiques, qui additionne les positifs valables et les négatifs valables. Elle apporte des correctifs pour le hasard et pour les valeurs maximales, s'il y a divergence entre le taux d'apparition dans le groupe étudié et le taux de base (Loeber et Dishion, 1983). De telles divergences sont courantes dans les études de prédiction de délinquance. L'indice ARH va de 0 à 100 , indiquant le pourcentage d'amélioration par rapport au hasard. Une valeur négative indique une relation inférieure à celle du hasard. Sa formule est :

$$
\begin{aligned}
& \text { ARH }=\% \text { d'amélioration sur le hasard } \times 100 \\
& \% \text { maximum de - \% de prédictions justes } \\
& \text { prédictions justes si le hasard joue seul }
\end{aligned}
$$


Dans les recherches où il $\mathrm{y}$ avait plus de deux catégories par variable, celles-ci étaient réduites à la dichotomie et ce, de manière à optimiser la concordance entre le taux de base et celui du groupe expérimental. On a préféré l'indice ARH aux autres mesures d'association parce qu'il est très indépendant de ces taux (Loeber et Dishion, 1983). On n'a pas calculé ARH dans les cas où l'un ou l'autre taux était très bas $(5 \%)$ vu que l'ARH n'est pas très stable aux extrémités de la distribution.

Avant de présenter les résultats, il faut faire quelques mises au point. Même s'il arrive qu'on ait des données relatives à des groupes, soit féminins, soit de race noire, la plupart des études portent sur des garçons de race blanche. D'où vient que nos conclusions s'appliquent surtout à cette population. De plus, les variables prédictives sont souvent mesurées par seulement quelques questions et avec peu de souci de répéter les tests ou d'établir la validité interne des instruments de mesure. Dans une moindre mesure, c'est aussi le cas des variables résultantes (mesures de délinquance). Néanmoins, la faiblesse des mesures est contrebalancée par la dimension relativement grande des échantillons et la multiplication des preuves à travers les recherches. En résumant plusieurs études, nous négligerons nécessairement les caractéristiques de chacune, comme le type d'échantillon, le type de répondant, l'intervalle de prédiction ou le contenu exact des échelles de comportement. Nous y gagnons toutefois une meilleure compréhension de la relation qui existe entre des prédicteurs spécifiques de délinquance et le comportement ultérieur.

Les tableaux des résultats présentent, les principales catégories de prédicteurs selon le sexe des sujets et selon les trois variables résultantes que sont la délinquance en général, la délinquance grave et la récidive. Tandis que dans la plupart des recherches, la délinquance générale comprend les délits statutaires, la délinquance grave se restreint habituellement aux délits contenus dans la partie I du FBI Crime Index, soit l'incendie criminel, le vol qualifié, le viol et l'homicide involontaire coupable. Faute d'espace, les données de prédiction, souvent réparties sur plusieurs années, sont seulement résumées ici. Pour le détail de ces recherches, prière de se référer au chapitre original duquel cet article est tiré (note 1). 


\section{LES PRÉDICTEURS COMPORTEMENTAUX}

Voyons d'abord les résultats relatifs aux prédicteurs comportementaux (tableau 1). Sur ces derniers, le présent compte rendu déborde le résumé précédemment réalisé par Loeber et Dishion (1983).

\section{L'AGRESSION}

Les études montrent une relation constante entre l'agressivité et la délinquance ultérieure. Pour la délinquance en générale, l'ARH médian était de $45,3 \%$ (variant de 16,4 à $51,4 \%$ ) dans les études portant exclusivement sur les garçons et de $25,2 \%$ ( 17 à $30,3 \%)$ dans les études de groupes mixtes. L'indice était un peu plus bas quand il s'agissait de prédire la récidive (médiane de $38,3 \%$; dispersion de 28,1 à $38,5 \%$ ). Notons que les recherches utilisaient tantôt les notations des instituteurs ou des groupes de pairs, tantôt l'autoévaluation, comme mesure d'agressivité, d'où on ne sait pas grand chose sur la portée prédictive de l'agressivité à domicile.

En moyenne, la proportion des faux négatifs était élevée, montrant qu'environ la moitié des jeunes qui s'avéraient délinquants n'avaient pas manifesté d'agressivité d'abord. Un taux élevé de faux négatifs n'est toutefois pas propre à l'agressivité dans le jeune âge. Nous verrons qu'il est aussi le fait d'autres prédicteurs. Nous reviendrons sur la signification de ce type d'erreur.

\section{LA CONSOMMATION DE DROGUE}

Nous n'avons trouvé que trois études reliant la consommation de drogue à la délinquance ultérieure. L'ARH médian pour la prédiction de la délinquance en général était de $53 \%$ (de 27 à $79 \%$ ). Ce comportement aidait aussi à prédire la récidive continue d'un petit groupe de garçons à l'agir délinquant déjà multiple (ARH de 42,6\%; Osborn et West, 1980). Contrairement aux autres prédicteurs, mesurés à la fin de l'enfance, la consommation de drogue l'a été seulement à la fin de l'adolescence, vraisemblablement à cause du fait que les jeunes enfants s'adonnent beaucoup moins à la drogue. Dans une seule recherche (Robins et al., 1970), on a fait la distinction entre les types de drogues. L'utilisation de drogues douces, étant plutôt 
TABLEAU 1

Résumé des comportements de l'enfance prédicteurs de la délinquance ultérieure

\begin{tabular}{|c|c|c|c|c|c|c|c|c|c|c|}
\hline \multirow[b]{2}{*}{ Prédicteur } & \multirow[b]{2}{*}{ Sexe } & \multicolumn{3}{|c|}{ Délinquance en général } & \multicolumn{3}{|c|}{ Délinquance grave } & \multicolumn{3}{|c|}{ Récidive } \\
\hline & & $n r^{2}$ & $\begin{array}{c}\text { ARH' }^{1} \\
\text { Median }\end{array}$ & Dispersion & $n r^{2}$ & $\begin{array}{c}\mathrm{ARH}^{1} \\
\text { Median }\end{array}$ & Dispersion & $n r^{2}$ & $\begin{array}{c}\text { ARH' } \\
\text { Median }\end{array}$ & Dispersion \\
\hline Agression & $\begin{array}{c}G \\
F+G\end{array}$ & $\begin{array}{l}5 \\
3\end{array}$ & $\begin{array}{l}45,3 \\
25,2\end{array}$ & $\begin{array}{l}16,4-51,4 \\
17,0-30,3\end{array}$ & & & & 3 & 38,3 & $28,1-38,5$ \\
\hline $\begin{array}{l}\text { Consommation } \\
\text { de drogue }\end{array}$ & G & 2 & 53,0 & $27,0-79,0$ & & & & 1 & 41,6 & \\
\hline $\begin{array}{l}\text { Absentéisme } \\
\text { scolaire }\end{array}$ & $\mathbf{G}$ & 4 & 25,5 & $20,4-26,3$ & 2 & 46,5 & $27,8-65,1$ & & & \\
\hline Mensonge & G & 2 & 22,4 & $22,0-22,7$ & & & & 1 & 31,0 & \\
\hline Vol & G & 2 & 37,4 & $17,1-57,8$ & 1 & 37,5 & & 1 & 60,6 & \\
\hline $\begin{array}{l}\text { Problèmes de } \\
\text { comportement }\end{array}$ & $\begin{array}{c}G \\
G \\
G+F\end{array}$ & $\begin{array}{r}12 \\
1 \\
2\end{array}$ & $\begin{array}{l}26,6 \\
82,9 \\
41,3\end{array}$ & $\begin{array}{r}4,0-64,2 \\
32,3-50,2\end{array}$ & 3 & 66,3 & $9,1-91,8$ & 3 & 52,5 & $26,0-89,0$ \\
\hline Réussite scolaire & G & 11 & 22,9 & $11,1-46,1$ & & & & 1 & 42,9 & \\
\hline $\begin{array}{l}\text { Échelles de } \\
\text { prédiction }\end{array}$ & G & 3 & 64,4 & $27,8-78,0$ & & & & 1 & 87,0 & \\
\hline Délinquance & $\underset{G+F}{G}$ & & & & & & & $\begin{array}{l}8 \\
2\end{array}$ & $\begin{array}{l}38,3 \\
35,1\end{array}$ & $\begin{array}{l}30,4-81,3 \\
33,6-36,5\end{array}$ \\
\hline
\end{tabular}

1. Amélioration par rapport au hasard.

2. Nombre de recherches. 
la norme pour certains groupes d'âge, ne peut être un prédicteur aussi puissant de délinquance que celle des drogues fortes.

Plusieurs études ont montré que maints délinquants persistants font une consommation polyvalente de drogues ou encore qu'ils consomment drogue et alcool (Petersilia et al., 1977; Tuchfield et al., 1982). Nous savons peu de choses sur la relation entre le début de la consommation et le début de la délinquance. L'utilisation de drogues apparaît-elle avant ou après la délinquance? Les données de Johnston et al. (1978), sont à l'effet que l'engagement délinquant était plus grand chez les consommateurs tardifs que chez les non-usagers. Cette étude a aussi montré que plus grave est la délinquance antérieure, plus grave est l'engagement subséquent dans la drogue. Une image populaire fait de l'enfoncement dans la délinquance un comportement destiné à entretenir une habitude de drogue. Mais nous ignorons pour combien de délinquants ce serait le cas.

\section{L'ABSENTÉISME SCOLAIRE}

Les quatre études qui ont mesuré l'absentéisme scolaire en tant que signal de délinquance ont obtenu un ARH médian de $25,5 \%$ (de 20,4 à 26,3\%). Cette variable était reliée à la délinquance grave ultérieure des enfants amenés à une clinique d'aide à l'enfance (ARH de 27,8\%; Nylander, 1979) comme des jeunes délinquants (ARH de 65,1\%; Glueck et Glueck, 1940). Dans cette dernière étude, $77,2 \%$ de tous les sujets faisaient l'école buissonnière, comparativement à une moyenne de $12,5 \%$ pour tous les sujets des échantillons non délinquants. Quant aux délinquants non absentéistes, ils se révèlent engagés dans une délinquance mineure plutôt que grave. On l'interprète par le temps libre, au cours des absences, riche en occasions de commettre des délits et de développer les habiletés et attitudes nécessaires à la poursuite d'une carrière criminelle.

\section{LE MENSONGE}

Le mensonge, qui accompagne souvent la carrière délinquante, est nécessaire pour dissimuler verbalement le comportement délinquant. Comme l'habileté à mentir est acquise plus jeune que la conduite délinquante, on croit qu'elle est un signe avant-coureur de la délinquance. La puissance prédictive du 
mensonge sur la délinquance ultérieure n'a toutefois pas été souvent mesurée. L'ARH médian des deux études reliant, par le biais de l'évaluation des parents ou des pairs, le fait de mentir à la délinquance officielle ultérieure était de $22,4 \%(22,0$ et $22,7 \%$ ). Mitchell et Rose (1981) ont trouvé que les enfants dits menteurs par leurs parents et instituteurs étaient les mêmes que les récidivistes identifiés dans un groupe de délinquants (ARH de $31 \%$ ).

\section{LE VOL}

Dans une large mesure, les faits rapportés étaient des vols simples ayant échappé à l'attention de la police. Il est prévisible que le vol au cours du jeune âge soit un bon prédicteur de délinquance, puisque les délits officiels consistent le plus souvent en atteintes aux biens. Seules deux études ont abordé le vol précoce comme signe avant-coureur de la délinquance en général et leur taux ARH diffère considérablement. L'étude de Mitchell et Rose (1981) sur les enfants-problèmes a décelé un taux de $57,8 \%$ contre seulement $17,1 \%$ dans la recherche de Nylander (1979) sur les pupilles d'une clinique. En contrepartie, selon cette dernière recherche, le chapardage et le vol simple permettaient aussi de prédire les crimes graves (ARH de 37,5\%). Les vols rapportés par les parents et les instituteurs étaient aussi des prédicteurs de récidive parmi un petit groupe de délinquants (ARH de 60,5\%; Mitchell et Rosa, 1981). Il est à noter que les sujets de ces études étaient déjà déviants sur certains plans et qu'on ignore si de tels larcins dans l'enfance garderaient leur qualité prédictive dans une population normale.

\section{LES PROBLÈMES DE COMPORTEMENT}

Tout un éventail de prédicteurs tels que le fait d'être qualifié d'antisocial ou de fauteur de troubles, une mauvaise socialisation, et des caractéristiques plus précises comme la destructivité, la témérité ou le fait de perturber la classe se sont vus attribuer un taux ARH médian de $30 \%$, pour prédire la délinquance générale des garçons. Néanmoins, certains s'écartaient beaucoup de la médiane. C'est ainsi que le fait pour un jeune d'être étiqueté d'antisocial se traduisait par une bien plus grande probabilité pour ce jeune d'être arrêté ultérieurement pour des délits étrangers à la circulation que pour son correspondant du 
groupe contrôle, avec un ARH de 64,2\% pour les garçons et de $82,9 \%$ chez les filles (pour une confirmation de la plus grande prévisibilité de la délinquance des filles, voir Werner, sous presse; Robins, sous presse). À l'autre extrême se situe l'hyperactivité, qu'on croit souvent fortement associée à la délinquance grave ultérieure (Hechtman et al., 1984; Mendelson et al., 1971) tandis qu'elle ne représente qu'un taux ARH de $4 \%$ (Nylander, 1979). Un point qui se révéla important fut celui de la concomitance de l'hyperactivité et de l'agressivité, avec un taux ARH de 45,3\% (Magnusson, 1984; voir aussi Schachar et al., 1981). Par rapport à la délinquance grave et à la récidive des garçons, les problèmes de comportement à un âge précoce ont produit des taux ARH médians de 66,3\% (variant de 9,2 à $91,8 \%$ ) et $52,5 \%$ (de 26 à $89 \%$ ) respectivement mais, ici comme antérieurement, l'hyperactivité s'est avérée un faible prédicteur (ARH de 9,2\%).

D'autres caractéristiques étudiées, qu'on ne peut qualifier de problèmes de comportement au sens strict, se rapportent à la situation économique des plus vieux adolescents. Farrington (1979) rapporte que l'instabilité au travail et le fait de n'avoir pas d'économies sont reliés à des condamnations ultérieures (ARH de 30 et $35,4 \%$ respectivement). Quand au chômage prolongé, il permettait de distinguer entre récidivistes persistants et temporaires (ARH de 28,1\%; Osborn et West, 1980).

\section{UN BAS NIVEAU DE REUSSITE SCOLAIRE OU DE Q.I.}

Dans maintes études comparatives, l'insuccès scolaire est révélé associé à la délinquance avec beaucoup de constance (Hirschi et Hindelang, 1977; Menard et Morse, 1984). Quant aux résultats des études longitudinales sur le succès scolaire et le quotient intellectuel comme prédicteurs de la délinquance ultérieure des garçons, ils comportent un ARH de 22,9\% (de 11,1 à $46,1 \%$ ). La plupart des études ont utilisé comme variable résultante la délinquance officielle. Toutefois, il s'est avéré que la pauvreté du vocabulaire vers dix ans et, plus tard, la faiblesse des résultats scolaires au moment de quitter les études étaient aussi reliées à une forte délinquance révélée (ARH de 27,4 et de $18,3 \%$ respectivement; Farrington, 1979). West et Farrington (1973) ont constaté un ARH de 42,9\% reliant le quotient intellectuel à la récidive. 
On ne sait pas bien quels sont les facteurs intermédiaires qui se placent entre le Q.I. ou le succès scolaire et la délinquance. Une école de pensée a désigné la réaction de l'enfant au système éducatif et à sa propre performance et considère l'échec éducatif comme un signe avant-coureur de délinquance (Hirschi et Hindelang, 1977). Selon une autre perspective, on considère davantage l'enfant comme victime des réactions institutionnelles spécifiques à un bas Q.I. et à de faibles performances scolaires (Hawkins et Doucek, sous presse; Menard et Morse, 1984). Une troisième hypothèse serait que les problèmes de comportement précoces précèdent l'insuccès.

À ce jour, un certain nombre d'études longitudinales appuient cette dernière hypothèse (McMichael, 1979; Stott, 1981; Spivack, 1983). Par exemple, Spinack, au terme d'une étude d'envergure, a conclu que, pour les deux sexes, la performance académique dans le jeune âge joue très peu et, lorsqu'elle joue, c'est de manière indirecte, à travers un comportement aberrant prédicteur et/ou une mauvaise conduite ultérieure, susceptible de mener à la délinquance. Dans la même veine, McMichael (1979) en est venu à la conclusion que les formes antisociales de la déviance scolaire sembleraient antérieures aux difficultés de lecture dans bon nombre de cas. Toutefois, les études portaient sur l'insuccès à l'école primaire. On n'y voit pas s'il en serait de même pour la délinquance au secondaire (Kirkegaard et al., 1977; Polk, 1975).

\section{LES INDICES DE PREDICTION}

On a tiré plusieurs indices de prédiction du comportement des enfants. Dans deux cas, on a retenu les prévisions de leurs instituteurs (Reckless et Dinitz, 1972; Scarpitti, 1964), qui se sont avérées utiles avec des taux d'ARH élevés $(64,4$ et 78 respectivement). Pour leur part, Stott et Wilson (1968) ont utilisé une échelle sur laquelle les instituteurs étaient appelés à situer leurs élèves par rapport au risque de délinquance, mais le taux $\mathrm{ARH}^{1}$ de ce score multiple ne fut que de $27,8 \%$. Enfin, Spivack (1983; Spivack et Cianci, sous presse) a élaboré un profil d'enfant à haut risque dès le début de l'école primaire, à partir

1. Voir page 5 . 
de comportements comme : le fait d'être surengagé socialement, l'impatience, l'effronterie, l'attitude de défi, le blâme d'autrui et des réactions inappropriées. Un score élevé à ce profil aidait à prédire la chronicité de l'agir délinquant, mais les faux-positifs s'avérèrent très nombreux. Nous reviendrons sur cette importante étude en abordant la prédiction de la délinquance chronique.

Il faut user d'une extrême prudence dans l'emploi de techniques prophétiques comme celles de Reckless et Dinitz (1972) ou de Scarpitti (1964). On ne peut avoir la certitude que les instituteurs ont basé leurs prédictions seulement sur le comportement des enfants, à l'exclusion d'autres facteurs comme le statut socio-économique, le comportement des frères et sœurs ou celui des parents. Cette critique s'applique jusqu'à un certain point à toutes les évaluations mais le risque s'accroît quand la tâche n'est pas bien spécifiée.

\section{LA DÉLINQUANCE PRÉCOCE}

Jusqu'à quel point la délinquance juvénile permet-elle de prédire la délinquance ultérieure? Étant donné la similitude entre le critère et son présumé prédicteur, la relation devrait être relativement serrée (Monahan, 1981). Plusieurs études se sont basées sur la délinquance révélée pour prédire tantôt la délinquance révélée continue, tantôt la récidive officielle. Le taux ARH médian pour leurs échantillons masculins était de $34,5 \%$ (variant de 30,4 à 41,2\%). Elliott et al. (1983) ont pour leur part utilisé la délinquance révélée à un moment pour en prédire la persistance, chez les garçons et les filles, sur une période de deux ans. Le taux ARH était de 33,6\% et la plupart des erreurs étaient des faux positifs, soit des jeunes qui avaient abandonné ou réduit leur engagement délinquant. Cinq recherches portant sur la délinquance officielle en tant que prédicteur de récidive ont produit un taux ARH médian de 45,5\% avec une variation de 32 à $81,3 \%$.

Les opinions sont partagées sur la question de la gravité des délits comme prédicteur de leur chronicité éventuelle. Par exemple, Elliott et al. (1983) ont conclu que la gravité du délit ayant conduit à l'arrestation ne permettait pas de prédire l'allure de la carrière. D'autres chercheurs ont abondé dans le même sens, comme Holland et McGarney (1984), Wolfgang et al. (1972). Mais certains autres ont trouvé que la gravité des délits 
aidait à prédire davantage l'engagement délinquant continu que l'engagement temporaire (Knight et West, 1975; Shannon, 1978). Pour Knight et West, le fait d'être, à 17 ans, trouvé coupable d'un délit grave était un puissant prédicteur d'une future condamnation, au début de l'âge adulte, avec un taux ARH de $81,3 \%$. Le taux d'erreurs était remarquablement bas, soit $16,7 \%$ de faux positifs et $6,3 \%$ de faux négatifs. Toutefois, dans une analyse subséquente des mêmes données, la gravité du premier délit de l'enfance s'est avérée un piètre prédicteur de l'agir délinquant chronique (Blumstein et al., 1985). Shannon (1978), utilisant l'analyse de régression multiple pour prédire la gravité de la délinquance, a trouvé que parmi toutes les variables, c'était les scores de gravité obtenus de 6 à 17 ans qui contribuaient le plus à la prédiction de la gravité des délits commis de 18 à 20 ans. À son tour, la gravité à cette dernière période était le meilleur prédicteur de la délinquance ultérieure.

Une première arrestation, tôt dans l'adolescence ou encore dans l'enfance, est-elle un meilleur prédicteur des délits futurs qu'une arrestation à la fin de l'adolescence? Farrington (1983) a trouvé que les 23 jeunes qui étaient devenus délinquants chroniques (trouvés coupables 6 fois et plus) vers 25 ans avaient tous antérieurement été condamnés au moins une fois autour de 15 ans. Ou, d'un autre point de vue, $32,3 \%$ de ceux qui avaient été trouvés coupables avant cet âge sont devenus des délinquants chroniques, contre aucun de ceux qui n'ont été condamnés qu'après 15 ans.

Robins et Radcliff (1978-1979) ont également constaté que l'arrestation avant 15 ans permettait de prédire la commission de délits à l'âge adulte (taux ARH de 36,5\%). Cette capacité prédictive repose probablement sur le fait que ceux qui commencent jeunes commettent davantage de délits et auront plus de problèmes de comportement que ceux qui commencent plus tard (Farrington, 1982; Loeber, 1982).

\section{UN BILAN SUR LES PRÉDICTEURS COMPORTEMENTAUX}

En résumant les données du tableau 1, il faut garder à l'esprit que les études que l'on a comparées sont fort différentes par l'échantillonnage, les prédicteurs, les critères et l'âge au moment des évaluations. En second lieu, il y a souvent une 
grande dispersion des valeurs pour chaque catégorie cependant que le nombre des recherches est habituellement restreint. D'où les conclusions sont, dans les meilleurs cas, provisoires. Comme il apparait au tableau 1, presque toutes les catégories de comportement aidaient davantage à prédire la délinquance grave ou la récidive que l'agir délinquant en général. Pour cette dernière, le taux ARH le plus élevé était de $27 \%$ (de 4 à $79 \%$ ), comparé à $65,7 \%$ (de 9,1 à $91,8 \%$ ) et à $38,5 \%$ (de 26 à $89 \%$ ) pour la gravité et la récidive respectivement. Il apparaît aussi que chez les garçons, l'agressivité (ARH médian de $45,3 \%$ ), le vol $(37,4 \%$ ) et la consommation de drogue ( $53 \%$ ) étaient de plus puissants prédicteurs que l'absentéisme scolaire ou le mensonge (ARH médians de 25,5 et $22,4 \%$ respectivement). Cette conclusion va dans le sens des découvertes de Robins (1966) à l'effet que les garçons amenés en clinique suite à un vol ou, dans une moindre mesure, à une agression, étaient plus susceptibles d'être éventuellement étiquetés sociopathes que ceux qui y étaient amenés pour toute autre raison. Pour l'ensemble des recherches, la délinquance féminine n'était en moyenne ni plus ni moins prévisible que celle des garçons. Dans quelques cas, elle l'était davantage.

En matière de récidive, on a curieusement constaté que les antécédents délinquants n'étaient pas meilleurs prédicteurs que le vol, les problèmes de comportement, la consommation de drogues et l'agression. Quant aux échelles de prédiction, qu'elles soient le fruit de jugements portés par les instituteurs ou d'une combinaison de variables, elles se sont avérées plus performantes que les variables prédictives isolées. Dans le tableau 1, les prédicteurs ont été dichotomisés pour raison de commodité, mais les études qui comportaient un plus grand nombre de catégories ont montré l'existence de stabilité comportementale chez les individus. Lorsqu'elles ont comparé trois groupes ordonnés du plus au moins déviant, invariablement, les études ont démontré que les deux groupes opposés étaient les plus susceptibles de maintenir leur position sur le continuum déviance-non déviance, tandis que le groupe intermédiaire se déplaçait davantage (Cline, 1980; Edelbrock et Loeber, 1985; Ghodsian et al., 1980; Loeber, 1982). Cela va dans le sens des études de personnalité qui ont montré que, dans une population, la constance à travers le temps n'est pas la même pour tous; que certains sont plus constants que d'autres (Bem et Allen, 1974). 


\section{LES PRÉDICTEURS CIRCONSTANCIELS}

On appelle ici variables circonstancielles les conditions de vie de l'enfant. Les détails des études longitudinales sur les prédicteurs circonstanciels ayant fait l'objet d'une recension récente (Loeber et Stouhamer-Loeber, sous presse), je me contenterai de les résumer (tableau 2).

\section{LES VARIABLES FAMILIALES SIMPLES}

La plupart des variables circonstancielles qui ont été étudiées relèvent de la famille. Le tableau 2 résume les résultats des recherches reliant les variables familiales à la délinquance future. Pour chaque variable, il présente le taux ARH médian et le nombre d'analyses sur lequel le taux est basé. Encore une fois, il faut interpréter les résultats avec circonspection vu le petit nombre des études pour chaque catégorie résultante (délinquance générale, délinquance grave, récidive). Les quatre premières variables (manque de surveillance, piètre discipline, peu d'engagement des parents et rejet de l'enfant) représentent des interactions parents-enfants. Parmi elles, le manque de surveillance semble le meilleur prédicteur de la délinquance en général (ARH de 21,1 à 80,8\%), mais c'est peut-être à cause de la rareté des comparaisons. Le manque d'engagement des parents vis-àvis de leur enfant de même que leur rejet de ce dernier améliorent modérément la prédiction, tandis que le manque de discipline est le plus faible prédicteur (ARH de 17,6 à 22,6\%). Mais c'est vraisemblablement à cause de la difficulté notoire inhérente à la mesure de la discipline (Patterson et Stouthamer-Loeber, 1984).

La pauvreté des relations conjugales est un prédicteur relativement puissant de la délinquance grave des enfants (ARH de 26,6 à $42,6 \%$ ) La criminalité et l'agressivité des parents, pour leur part, améliorent davantage la prédiction de la délinquance en général (ARH médian de $31 \%$ ) que de la délinquance grave $(19,5 \%)$. En comparaison, l'absence des parents est plus faible comme prédicteur de la délinquance générale tant dans les échantillons masculins (ARH médian de $20,8 \%$ ) que mixtes $(18,6 \%)$. Le mauvais état de santé des parents s'avère aussi un faible prédicteur (ARH médian de 14,2\%).

\section{LES VARIABLES FAMILIALES MULTIPLES}

Les prédicteurs élaborés à partir d'une combinaison de variables familiales sont habituellement plus performants que 
ceux qui consistent en une variable simple. Les Glueck (1950) ont introduit la prédiction à partir de facteurs multiples lorsqu'ils ont construit une table à partir principalement des variables d'interactions comme la discipline, la surveillance et l'affection. Depuis, des chercheurs successifs ont modifié leur table. Même si les études de ce genre ont été critiquées à maints égards (Prigmore, 1963; Reiss, 1951), elles ont constamment montré que les pratiques éducatives des parents contribuaient grandement à la prédiction de la délinquance des enfants. Un autre ensemble de recherches a utilisé des combinaisons de variables familiales et comportementales (McCord, 1979; Osborn et West, 1978; Wadsworth, 1979; West et Farrington, 1973; Farrington et Tarling, 1985). Leurs résultats furent à peu près les mêmes : un taux ARH médian de $56,4 \%$ pour la prédiction de la délinquance générale des échantillons de garçons et de $58,5 \%$ pour les échantillons mixtes; un taux de $72,3 \%$ pour la prédiction de la récidive. Cela dénote une amélioration substantielle sur les taux ARH médians des variables simples.

\section{LE STATUT SOCIO-ÉCONOMIQUE}

Il existe un bon nombre de revues de littérature sur les relations entre la classe sociale et la délinquance (Braithwaite, 1980; Hindelang et al., 1979; Title et al., 1978). La plus exhaustive est celle de Braithwaite qui conclut que la classe sociale aide à prédire la délinquance officielle et la délinquance révélée grave et, dans une moindre mesure, la délinquance révélée en général. La plupart des études recensées relèvent toutes les variables simultanément, de sorte qu'elles ne montrent pas une relation prédictive. Néanmoins, le statut socio-économique est relativement stable, d'où on peut déduire qu'il a précédé l'agir délinquant. Loeber et Dishion (1983) ont pour leur part fait la recension des études longitudinales sur la question. Le statut socio-économique, comme la plupart des autres variables du contexte familial entretient une relation prédictive plutôt faible avec la délinquance en général (ARH médian de 17,7\%) et avec la récidive (14\%). D'autre part, il est prouvé, à partir des études de délinquance révélée, que les basses strates socio-économiques sont surreprésentées chez les délinquants chroniques (Elliott et Huizinga, sous presse). 


\section{LES PAIRS}

Nous n'avons réussi à trouver qu'une étude longitudinale relative à l'influence des pairs déviants sur la délinquance. Farrington (1979) a trouvé que la fréquentation de pairs antisociaux à 18 ans présentait un taux ARH de 32,5\%. Comme l'influence des amis déviants joue un rôle central dans plusieurs théories de la délinquance, il est étonnant qu'on n'ait pas bien clarifié la question de ses relations temporelles avec la délinquance (mais voir Elliott et al., 1983)

\section{COMPARAISON ENTRE LES PRÉDICTEURS COMPORTEMENTAUX ET CIRCONSTANCIELS}

On a généralement cru que les variables comportementales avaient plus d'impact prédictif que les variables circonstancielles (Monahan, 1981). Une comparaison entre nos deux tableaux le confirme. Le comportement de l'enfant permet de mieux prédire sa délinquance ultérieure que des variables contextuelles comme l'absence des parents, leur santé ou leur statut socio-économique. Toutefois, plusieurs variables d'interactions comme le manque de surveillance ou le rejet ont tendance à être également de puissants prédicteurs.

L'utilisation des problèmes de comportement individuels de l'enfant, comme celle des variables familiales, a permis d'améliorer la prédiction de la délinquance de 25 à $35 \%$. Bien que ce soit considérable, il y a encore une grande marge d'erreurs. Par contre, la combinaison de variables par le biais d'échelles de prédiction ou de scores constitués à partir de plusieurs variables familiales s'est avérée plus performante en doublant l'amélioration qu'avaient engendrée les variables prises une à une.

\section{L'IDENTIFICATION PRÉCOCE DE LA DÉLINQUANCE CHRONIQUE}

Trois recherches sur les délinquants persistants s'accordent à dire que ces sujets sont reconnaissables avant même l'école secondaire. Comme nous l'avons mentionné à propos de l'étude de Spivack (1983), les futurs délinquants chroniques (4 délits ou plus) se distinguaient à l'école primaire par le surengagement social, l'impatience, l'effronterie, une attitude de défi, le blâme 
d'autrui et des réactions impertinentes. Le moment optimal pour les identifier était la deuxième année primaire mais $68 \%$ des futurs délinquants chroniques étaient déjà reconnaissables à l'école maternelle. D'après les analyses de Farrington (Blumstein et al., 1985), les délinquants persistants (six délits ou plus à 25 ans) avaient, à treize ans ou même avant, des scores élevés sur trois des sept facteurs suivants : le fait d'être un fauteur de troubles, la désorganisation, l'acting-out, le handicap social, la criminalité des parents, la mauvaise éducation et un faible quotient intellectuel. Ces deux études ont en commun des taux relativement bas de faux négatifs soit $4,5 \%$ et $13 \%$. Une troisième étude, de Craig et Glick (1968) a aussi produit relativement peu de faux positifs $(20,5 \%)$. Cette recherche qui mesurait les problèmes de comportement des enfants de la première à la troisième année y décelait une grande valeur prédictive quant à la délinquance grave et/ou persistance (ARH de 66,3\%).

Ces trois recherches nous indiquent que les patterns de comportement précoces des deux tiers - sinon de presque tous les délinquants persistants - sont reconnaissables dès l'âge de l'école élémentaire. Cette conclusion corrobore les résultats de Robins (1966) à l'effet que quatre enfants antisociaux sur cinq amenés à une clinique sur la question des délinquants chroniques et les travaux de Robins (1966) se rejoignent pour dire que la conduite antisociale grave surgit rarement du néant à l'âge adulte, mais qu'elle a commencé bien plus tôt (Robins et Radcliff, 1978-1979).

Il faut remarquer, dans ces recherches, le grand nombre d'erreurs de faux positifs $(84,1,78,4$ et $66,7 \%$ respectivement). Il signifie ou bien que beaucoup ont abandonné la délinquance avant qu'on puisse la qualifier de chronique ou encore que plusieurs n'ont pas été pris par la police. Une autre hypothèse est qu'on aurait décelé plus de délinquants chroniques si la période de follow-up avait été plus longue. Mais pour le moment, un taux élevé de faux positifs affaiblit l'outil de tamisage que peut constituer un prédicteur. Néanmoins, les raisons en faveur de l'intervention précoce demeurent même s'il n'y a pas de tamisage. Le plus important programme d'intervention précoce réalisé à ce jour est le Perry Preschool Program en vertu duquel les enfants d'âge préscolaire provenant de milieux défavorisés furent répartis au hasard entre un groupe expérimental et un groupe contrôle (Berrueta-Clement, Schweinhart et Barnett, 
TABLEAU 1

Résumé des comportements de l'enfance prédicteurs de la délinquance ultérieure

\begin{tabular}{|c|c|c|c|c|c|c|c|c|c|c|}
\hline \multirow[b]{2}{*}{ Prédicteur } & \multirow[b]{2}{*}{ Sexe } & \multicolumn{3}{|c|}{ Délinquance en général } & \multicolumn{3}{|c|}{ Délinquance grave } & \multicolumn{3}{|c|}{ Récidive } \\
\hline & & $n r^{2}$ & $\begin{array}{l}\text { ARH } \\
\text { Médian }\end{array}$ & Dispersion & $n r^{2}$ & $\begin{array}{l}\text { ARH }{ }^{1} \\
\text { Médian }\end{array}$ & Dispersion & $\mathrm{nr}^{2}$ & $\begin{array}{l}\text { ARH' } \\
\text { Médian }\end{array}$ & Dispersion \\
\hline $\begin{array}{l}\text { Manque de } \\
\text { surveillance }\end{array}$ & $\mathrm{G}$ & 2 & 51,0 & $21,2-80,0$ & 1 & 29,2 & & & & \\
\hline $\begin{array}{l}\text { Désengagement } \\
\text { parents }\end{array}$ & $\mathrm{G}+\mathrm{F}$ & 1 & 31,0 & & & & & & & \\
\hline Piètre discipline & G & 1 & 22,6 & & 1 & 17,6 & & & & \\
\hline $\begin{array}{l}\text { Rejet par les } \\
\text { parents }\end{array}$ & G & & & & 2 & 38,7 & $33,7-43,8$ & & & \\
\hline $\begin{array}{l}\text { Criminalité et } \\
\text { agressivité des } \\
\text { parents }\end{array}$ & $G$ & 2 & 31,0 & $24,4-37,6$ & 5 & 19,5 & $10,8-34,4$ & 1 & 26,4 & \\
\hline $\begin{array}{l}\text { Problèmes } \\
\text { conjugaux }\end{array}$ & G & & & & 2 & 34,4 & $26,2-42,6$ & & & \\
\hline $\begin{array}{l}\text { Absence des } \\
\text { parents }\end{array}$ & $\underset{G+F}{G}$ & $\begin{array}{l}2 \\
3\end{array}$ & $\begin{array}{l}20,8 \\
18,6\end{array}$ & $\begin{array}{r}11,5-30,4 \\
7,4-25,1\end{array}$ & 1 & 8,5 & & & & \\
\hline $\begin{array}{l}\text { Mauvaise santé } \\
\text { des parents }\end{array}$ & $\mathrm{G}$ & 6 & 14,2 & $5,7-22,9$ & & & & & & \\
\hline $\begin{array}{l}\text { Handicaps } \\
\text { familiaux multiples }\end{array}$ & $\underset{G+F}{G}$ & $\begin{array}{l}5 \\
3\end{array}$ & $\begin{array}{l}56,4 \\
58,4\end{array}$ & $\begin{array}{l}45,7-86,7 \\
49,5-86,7\end{array}$ & & & & 3 & 72,3 & $46,9-81,9$ \\
\hline $\begin{array}{l}\text { Statut socio- } \\
\text { économique }\end{array}$ & G & 6 & 17,7 & $10,5-30,9$ & & & & 3 & 14,0 & $11,7-49,3$ \\
\hline Pairs déviants & $\mathrm{G}$ & 1 & 32,5 & & & & & & & \\
\hline
\end{tabular}

1. Amélioration par rapport au hasard.

2. Nombre de recherches. 
1984). Parvenus aux âges de 19 à 24 ans, les sujets du groupe expérimental avaient fait l'objet de 73 arrestations au total, comparés à environ le double dans le groupe contrôle. La dimension des groupes échantillons rend difficile la démonstration statistique de l'influence du programme sur la délinquance chronique, mais les résultats vont certainement dans le bon sens.

Outre le début précoce, les résultats présentés font apparaître plusieurs dimensions du comportement de l'enfance de la plus haute importance pour l'identification des cas à haut risque de délinquance chronique (Loeber, 1982). La première est que plusieurs de ces enfants manifestent une variété de problèmes de comportement plutôt qu'un seul (cf. Loeber, 1982; Robins et Radcliff, 1978-1979). Si on en croit les autres recherches, il est probable que ces jeunes présentent plusieurs configurations de tels problèmes, tandis que ceux qui s'en tiennent à une seule sont moins enclins à l'agir délinquant ou à d'autres inadaptations (Loeber, 1982; McGee et al., 1983; Mitchell et Rosa, 1981; Schachar et al., 1981).

\section{CONCLUSION}

Voici les conclusions majeures de notre recension des prédicteurs de la délinquance :

1) Les problèmes de conduite précoces - agression, vol, absentéisme scolaire, mensonge, consommation de drogue ne permettent pas seulement de prédire la délinquance générale qui apparaîtra plusieurs années plus tard mais particulièrement la délinquance grave et, dans certains cas, la récidive. En fait, ces résultats se répètent immanquablement d'une étude à l'autre, sur des sujets échantillonnés en divers lieux et pays. De plus, ils s'appliquent aux filles comme aux garçons bien qu'il y ait moins de recherches sur les premières.

2) Le fait d'être arrêté ou reconnu coupable comme mineur est un prédicteur modérément bon des mêmes événements à l'âge adulte; la gravité d'un délit de l'enfance semble un bon signe avant-coureur de la délinquance persistante et grave à l'âge adulte.

3) Les variables familiales individuelles aidaient modérément à prédire la délinquance ultérieure des enfants. Une mau- 
vaise surveillance exercée par les parents et le rejet de l'enfant se sont avérés des prédicteurs particulièrement puissants, tandis que les autres variables relatives à l'éducation des enfants comme le manque de discipline et le manque d'engagement étaient légèrement moins forts. De plus, la criminalité et l'agressivité des parents, tout comme le désaccord conjugal, étaient modérément bons prédicteurs. L'absence des parents, leur santé et le statut socio-économique étaient de plus faibles indicateurs de la délinquance future. Les meilleurs prédicteurs de tous étaient les handicaps familiaux multiples.

4) Les mauvais résultats scolaires sont jusqu'à un certain point annonciateurs de délinquance future mais tout nous porte à croire que leur effet se produit par l'intermédiaire des problèmes de comportement qui l'accompagnent.

5) La majorité des délinquants chroniques éventuels peuvent être reconnus par leurs problèmes de conduite et autres handicaps dès l'âge de l'école primaire.

Les résultats de notre recension des prédicteurs doivent être interprétés avec prudence. Premièrement, le petit nombre d'études portant sur chaque catégorie de prédicteurs rend hasardeuses les généralisations aux autres populations. Deuxièmement, comme on a considéré aussi bien les études rétrospectives que prospectives, leurs résultats ne sont pas immédiatement transférables aux futures recherches prospectives. Troisièmement, parmi les études recensées, certaines se rapportaient à des groupes particuliers d'enfants comme, par exemple, les enfants admis à une clinique, les auteurs d'un premier délit ou encore désignés d'une autre manière comme déviants. Or, la capacité prédictive des signes avant-coureurs a tendance à être plus élevée dans ces groupes que lorsqu'ils sont appliqués à une population normale. Quatrièmement, on compare l'efficacité des prédicteurs en prenant comme critère l'amélioration relative sur le hasard. On ignore toujours si l'ordre des prédicteurs serait le même si on utilisait d'autres indices de capacité prédictive, comme par exemple l'estimé du coût moyen (Duncan et al., 1953; Simon, 1971). Cinquièmement, nous manquons de recherches sur la prédiction de la délinquance révélée. Dans celle de Cambridge sur le développement de la délinquance, on a trouvé que les condamnations étaient plus prévisibles que la délinquance révélée parce que les signes avant-coureurs des 
premières étaient plus nombreux que ceux de la seconde (Farrington et Tarling, 1985).

Malheureusement, nous n'avons relevé qu'une seule étude tentant de prédire l'engagement dans la délinquance grave révélée par questionnaire (Elliott et al., 1983). Sixièmement, même si les résultats des études de prédiction se maintiennent lors de répliques dans d'autres configurations de populations, il persiste un manque considérable d'informations relatives aux facteurs de risque dans des îlots de population. Notamment, il y a une curieuse pénurie de recherches dans la communauté noire et d'autres minorités. Enfin, faut-il souligner que les signes avant-coureurs de délinquance ne peuvent être assimilés à des facteurs de causalité tant qu'on n'a pas de preuve de la direction de l'influence causale. Par exemple, si on postule que l'incapacité des parents est à la base des problèmes de conduite ou de la délinquance des enfants, on ne saurait exclure la possibilité que tous les parents aient du mal à élever un enfant au tempérament difficile.

Nous avons mentionné qu'une combinaison de bons prédicteurs, tend à produire une amélioration cumulative de l'efficacité d'un outil de tamisage (Robins et Wish, 1977; Rutter, 1978). Il existe plusieurs méthodes pour optimiser la combinaison des prédicteurs comme celles de Burgess, Glueck et les autres (Farrington et Tarling, 1985; Simon, 1971). Les comparaisons plus sophistiquées qui attribuent des poids particuliers à chaque élément, comme la régression multiple, ont montré que la simple sommation a tendance à moins perdre de puissance lors des répliques (Farrington et Tarling, 1985).

Copas et Tarling (1984) ont montré comment calculer la perte de puissance dans ces études prédictives. Farrington (Farrington et Tarling, 1985), après avoir recensé les prédicteurs de délinquance a conclu qu'on n'améliorera pas la prédiction par l'invention et l'utilisation de méthodes statistiques plus sophistiquées pour sélectionner et combiner les variables dans un instrument de prédiction, du moins avec les méthodes de mesures actuelles. Il mise plutôt sur le développement d'instruments de mesures plus valides, fidèles et sensibles. À cela nous ajoutons l'identification de meilleurs prédicteurs. On pourrait y arriver en mettant l'accent sur la détection tantôt des vrais positifs, tantôt des vrais négatifs (ceux qui ne deviendront pas 
délinquants), et tantôt sur les faux positifs (ceux qui abandonnent les comportements problèmes).

Si la construction de facteurs multiples s'avère utile pour l'identification de groupes de jeunes à haut risque de délinquance, parfois, nous l'avons souligné, le processus délinquant se dessine mieux à l'aide de prévisions successives que par la combinaison de tous les prédicteurs les plus puissants au temps 1 et leur relation avec une résultante dans un futur passablement lointain. Par contre, il ne faut pas oublier que l'impact de certains prédicteurs (comme le manque de socialisation dans la famille) s'accroît vraisemblablement à mesure que se prolonge l'exposition de l'enfant aux circonstances défavorables. C'est ainsi qu'une lacune de quelques mois dans la surveillance peutêtre un moins bon prédicteur que si elle dure plusieurs années.

Dans les faits, toutes les recherches que nous avons recensées référaient à un modèle de passage à l'acte plutôt qu'à un modèle d'aptitude. Nous croyons pour notre part qu'un comportement délinquant bien ancré est le reflet d'une aptitude; qu'il y a d'abord eu acquisition d'habiletés. Comme exemples d'habiletés, mentionnons celle de se renseigner sur les bonnes affaires, de s'adjoindre des partenaires, de planifier un crime, de changer son identité, sans parler de celles d'ouvrir des coffres et de manigancer des fraudes, etc. (Petersilia et al., 1977). Dans un modèle de passage à l'acte, il s'agit de savoir si un comportement se produit ou non tandis que dans un modèle d'aptitude, il faut voir s'il y a eu apprentissage de comportements quelque part dans le temps et quels sont les facteurs qui déclenchent ou non le passage à l'acte. L'étude de la délinquance est pour une bonne part celle du passage à l'acte et elle cherche rarement à savoir si des aptitudes acquises tôt auraient quelque chose à voir avec le fait d'enfreindre la loi, même seulement beaucoup plus tard. C'est peut-être l'exercice dans le jeune âge de la tricherie, de la brutalité ou de la manigance qui compte. Les découvertes de Chaiken et Chaiken (1984) et d'Osborn et West (1980) s'inscrivent dans la même voie. Après avoir interviewé des prévenus incarcérés et un échantillon d'hommes dans la communauté, ils ont noté que certains individus se réengageaient dans des activités délinquantes après des intervalles de deux à cinq ans sans crime. Les théories de la délinquance doivent être capables de rendre compte aussi bien des aptitudes que des raisons pour lesquelles il y a passage à l'acte dans certains cas et non dans d'autres. 


\section{Notes}

1. Il faut signaler que dans l'étude de Magnusson, Slottin et Duner (1983), le vol qualifié était classé dans les crimes contre la propriété.

2. Kraus (1973), dans une étude portant sur les comportements problèmes d'enfants jusqu'en 6e année, découvrit que tous les enfants problèmes, sauf trois, avaient déjà été identifiés comme tels à la fin de la $3^{\text {e }}$ année. Était considéré enfant problème celui qui recevait une évaluation élevée de trois professeurs différents à partir du jardin d'enfant. Dans cette recherche, on n'a pas mis ces évaluations en relation avec la délinquance subséquente.

\section{Remerciements}

Ce chapitre est une révision partielle d'un texte rédigé par l'auteur senior et Tom Dishion portant sur la prédiction de la délinquance chez les garçons et publiée dans le Psychological Bulletin de 1983. Il a été écrit alors que l'auteur senior était membre du comité de la recherche sur les carrières criminelles du «National Academy of Science». Il a trouvé une source d'inspiration chez les collègues qui y siégeaient. Les auteurs sont reconnaissants à David Farrington et à Charles Lidz pour leurs commentaires sur une version préliminaire de ce texte. Danette Waller a contribué à l'analyse des données alors que Celio Eatman a aidé à la préparation de l'article.

\section{RÉFÉRENCES}

BELL, R.Q. et D. PEARL (1982), «Psychosocial Change in Risk Groups : Implications for Early Identification", Journal of Prevention in Human Services, 1, 45-59.

BEM, D.J. et A. ALLEN (1974), "On Predicting some of the People some of the Time : The Search for Cross-Situational Consistencies in Behavior", Psychological Review, 81, 506-520.

BERRUETA-CLEMENTS, J.R., L.J. SCHWEINERHART, W.S. BARNETT, E.S. EPSTEIN et D.P. WEIKART (1984), Changed Lives : The Effects of the Perry Preschool Program on Youths Through Age 19, Ypsilanti, MI, High/Scope Press. 
BLUMSTEIN, A., D.P. FARRINGTON et S. MOITRA (1985), "Delinquency Careers : Innocents, Amateurs, and Persisters», dans M. Tonry et N. Morris (édit.), Crime and Justice (vol. 6), Chicago, University of Chicago Press.

BRAITHWAITE, J. (1981), "The Myth of Social Class and Criminality Reconsidered», American Sociological Review, 46, 36-57.

CHAIKEN, M.R. et J.M. CHAIKEN (1984), "Offender Types and Public Policy», Crime and Delinquency, 30, 195-226.

CLINE, H.F. (1980), "Criminal Behavior over the Life Span», dans O.G. Brim et J. Kagan (édit.), Constancy and Change in Human Development, Cambridge, MS, Harvard University Press.

COPAS, J.B. et R. TARLING (1984), Some Methodological Issues in Making Predictions, Paper presented at the National Academy of Sciences panel on Research on Criminal Careers, Woods Hole, MS.

CRAIG, M.M. et S.J. GLICK (1968), «School Behavior Related to Later Delinquency and Nondelinquẹncy», Criminologica, 5, 17-27.

DUNCAN, O.D., L.E. OHLIN, A.J. REISS et H.R. STANTON (1953), "Formal Devices for Making Selection Decisions", American Journal of Sociology, 58, 573-584.

EDELBROCK, C. et R. LOEBER (1985), The Development of Antisocial Behavior in Childhood and Adolescence, Unpublished manuscript, University of Massachussetts Medical School, Worcester, MS.

ELLIOTT, D.S., F.W. DUNFORD et D. HUIZINGA (1983), The Identification and Prediction of Career Offenders Utilizing Self-Reported and Official Data, Unpublished manuscript, Behavioral Research Institute, Boulder, CO.

EMMERICH, W. (1964), "Continuity and Stability in Early Social Development», Child Development, 35, 311-332.

FARRINGTON, D.P. (1976), Statistical Prediction Methods in Criminology, Paper presented at the NATO Advanced Institute on computer Assisted Decision Making in Parole, Cambridge, England.

FARRINGTON, D.P. (1979), «Environmental Stress, Delinquent Behavior, and Convictions", dans I.G. Sarason et D.C. Spielberger (édit.), Stress and Anxiety (vol. 6), Washington, Hemisphere.

FARRINGTON, D.P. (1983), "Delinquency from 10 to 25 », dans K.T. Van Dusen et S.A. Mednick (édit.), Antecedents of Agression and Antisocial Behavior, Boston, Kluwer-Nijhoff.

FARRINGTON, D.P. et R. Tarling (édit.) (1985), Prediction in Criminology, Albany, NY, SUNY Press.

GLUECK, S. et E.T. GLUECK (1940), Juvenile Delinquents Grow Up, New York, Commonwealth Fund.

GLUECK, S. et E.T. GLUECK (1950), Unraveling Juvenile Delinquency, Cambridge, MS, Harvard University Press.

GOTTFREDSON, S.D. et D.M. GOTTFREDSON (1984), Accuracy of Prediction Models, Paper presented to the National Research Council's Panel on 
Research on Criminal Careers, National Academy of Sciences, Woods Hole, MS.

GREENWOOD, P. et A. ABRAHAMSE (1982), Selective Incapacitation, Report to the National Institute of Justice, Santa Monica, CA, Rand Corporation.

HAWKINS, J.D. et H. DOUECK (sous presse), «Social Development and the Prevention of Antisocial Behavior among Low Achievers», dans D.H. Crowell (édit.), Childhood Aggression and Violence, New York, Plenum.

HECHTMAN, L., T. PERLMAN et R. AMSEL (1984), «Hyperactives as Young Adults - Initial Predictors of Adult Outcome», The Journal of the American Academy of Child Psychiatry, 23, 250-260.

HINDELANG, M.J., T. HIRSCHI et J.G. WEIS (1979), «Correlates of Delinquency: The Illusion of Discrepancy between Self-Report and Official Measures», American Sociological Review, 44, 995-1014.

HIRSCHI, T. et M.J. HINDELANG (1977), «Intelligence and Delinquency : a Revisionist Review», American Sociological Review, 42, 571-587.

HOLLAND, T.R. et B. McGARVEY (1984), «Crime Specialization, Seriousness, Progression, and Markov Chains", Journal of Consulting and Clinical Psychology, 52, 837-840.

JOHNSTON, L.D., P.M. O'MALLEY et L.K. EVELAND (1978), „Drugs and Delinquency : A Search for Causal Connections», dans D.B. Kandel (édit.), Longitudinal Research on Drug Use, New York, Wiley.

KAGAN, J. (1971), Change and Continuity in Infancy, New York, Wiley.

KIRKEGAARD-SORENSON, L. et S.A. MEDNICK (1977), "A Prospective Study of Predictors of Criminality", dans S.A. Mednick et K.O. Christiansen (édit.), Biosocial Bases of Criminal Behavior, New York, Gardner Press.

KNIGHT, B.J. et D.J. WEST (1975), «Temporary and Continuing Delinquency», British Journal of Criminology, 15, 43-50.

KRAUS, P.E. (1973), Yesterday's Children : A Longitudinal Study of Children from Kindergarten into Adult Years, New York, Wiley.

LEMERT, E.M. (1951), Social Pathology, New York, McGraw-Hill.

LOEBER, R. (1982), "The Stability of Antisocial and Delinquent Child Behavior : A Review», Child Development, 53, 1431-1446.

LOEBER, R. et T.J. DISHION (1983), «Early Predictors of Male Delinquency : A Review», Psychological Bulletin, 94, 68-99.

LOEBER, R., T.J. DISHION et G.R. PATTERSON (1984), «Multiple Gating : A Multistage Assessment Procedure for Identifying Youths at Risk for Delinquency», Journal of Research on Crime and Delinquency, 21, 7-32.

LOEBER, R. et M. STOUTHAMER-LOEBER (sous presse), «Models and Meta-Analyses of the Relationships between Family Variables and Juvenile Conduct Problems and delinquency", dans N. Morris et M. Tonry (édit.), Crime and Justice: An Annual Review of Research, (vol. 7), Chicago, University of Chicago Press. 
McGEE, R., P.A. SILVA et S. WILLIAMS (1983), «Parents' and Teachers' Perceptions of Behavior Problems in Seven-Year-Old Children», The Exceptional Child, 30, 151-161.

McMiCHAEL, P. (1979), "The Hen or the Egg? Which Comes First - Antisocial Emotional Disorders or Reading Disability?", British Journal of Educational Psychology, 49, 226-238.

MAGNUSSON, D. (1984), Early Conduct and Biological Factors in the Developmental Background of Adult Delinquency, Paper presented at Henry Tajfel Memorial Lecture, Oxford.

MAGNUSSON, D. (1985), Personal Communication.

MAGNUSSON, D., H. STOTTIN et A. DUNER (1983), "Agression and Criminality in a Longitudinal Perspective», dans K.T. Van dusen et S.A. Melnick (édit.), Antecedents of Aggression and Antisocial Beahvior, Boston, Kluwer-Nijhoff.

MENARD, S. et B.J. MORSE (1984), «A Structuralist Critique of the IQDelinquency Hypothesis: Theory and Evidence», American Journal of Sociology, 89, 1347-1378.

MENDELSON, W., N. JOHNSON et M.A. STEWART (1971), «Hyperactive Children as Teenagers. A Follow-Up Study", Journal of Child Psychology and Psychiatry, 22, 19-33.

MONAHAN, J.D. (1978), "The Prediction of Violence in Juveniles», dans The Serious Juvenile Offender, Washington, D.C., U.S. Department of Justice.

MONAHAN, J.D. (1981), Clinical Prediction of Violent Behavior, Washington, DC, U.S. Government Printing Office.

NYLANDER, I. (1979), "A 20-Year Prospective Follow-Up Study of 2164 Cases at the Child Guidance Clinics in Stockholm», Acta Paediatrica Scandinavica, 68 (suppl. 276), 1-45.

OLWEUS, D. (1979), «Stability of Aggressive Reaction Patterns in Males : A Review», Psychological Bulletin, 86, 852-857.

OSBORN, S.G. et D.J. WEST (1978), "The Effectiveness of Various Predictors of Criminal Careers", Journal of Adolescence , 1, 101-117 (authors' updated and corrected version).

OSBORN, S.G. et D.J. WEST (1980), «Do Young Delinquents Really Reform?», Journal of Adolescence, 3, 99-114.

PATTERSON, G.R. (1982), A Social Learning Approach, vol. 3 : Coercive Family Process, Eugene, Oregon, Castalia Publishing Company.

PATTERSON, G.R. et M. STOUTHAMER-LOEBER (1984), «The Correlation of Family Management Practices and Delinquency", Child Development, $55,1299-1307$.

PETERSILIA, J., P.W. GREENWOOD et M. LAVIN (1977), Criminal Careers of Habitual Felons, Santa Monica, CA, Rand Corporation.

POLK, K. (1975), "Schools and the Delinquency Experience", Criminal Justice and Behavior, 2, 315-338. 
PRIGMORE, C.S. (1963), «An Analysis of Rater Reliability of the Glueck Scale for the Prediction of Juvenile Delinquency", Journal of Criminal Law, Criminology and Police Science, 54, 30-41.

RECKLESS, W.C. et S. DINITZ (1972), The Prevention of Juvenile Delinquency, Columbus, $\mathrm{OH}$, Ohio State University Press.

REISS, A.J. (1951), «The Accuracy, Efficiency, and Validity of a Prediction Instrument", American Journal of Sociology, 56, 552-561.

ROBINS, L.N. (1966), Deviant Children Grow-Up: A Sociological and Psychiatric Study of Sociopathic Personality, Baltimore, Williams et Williams.

ROBINS, L.N. (sous presse), "Changes in Conduct Disorder Over Time», dans D.C. Farren et J.D. McKinney (édit.), Risk in Intellectual and Psychosocial Development, New York, Academic Press.

ROBINS, L.N., H.S. DARVISH et G.E. MURPHY (1970), "The Long-Term Outcome for Adolescent Drug Users : A Follow-Up Study of 76 Users and 146 Nonusers», dans J. Zubin et A.M. Freedman (édit.), The Psychopathy of Adolescence, New York, Grune et Stratton.

ROBINS, L.N. et K.S. RATCLIFF (1978-1979), «Risk Factors in the Continuation of Childhood Antisocial Behavior into Adulthood", International Journal of Mental Health, 7, 96-116.

ROBINS, L.N. et E. WISH (1977), "Childhood Deviance as a Developmental Process : A Study of 223 Urban Black Men from Birth to 18", Social Forces, 56, 448-473.

RUTTER, M. (1978), «Family, Area and School Influences in the Genesis of Conduct Disorders", dans L.A. Hersov, M. Berger et D. Shaffer (édit.), Aggression and Antisocial Behavior in Childhood and Adolescence, Oxford, Pergamon.

SCARPITTI, F.R. (1964), "Can Teachers Predict Delinquency?", The Elementary School Journal, 65, 130-136.

SCHACHAR, R., M. RUTTER et A. SMITH (1981), «The Characteristics of Situationally and Pervasively Hyperactive Children: Implications for Syndrome Definition», Journal of Child Psychology and Psychiatry, 22, 375-392.

SHANNON, J.W. (août 1978), A Cohort Study of the Relationship of Adult Criminal Careers to Juvenile Careers, Paper presented at the International Symposium on Selected Criminological Topics, University of Stockholm, Sweden.

SIMON, F.H. (1971), Prediction Methods in Criminology, London, HMSO.

SPIVACK, G. (1983), High Risk Early Behaviors Indicating Vulnerability to Delinquency in the Community and School - a 15-Year Longitudinal Study, Report to the Office of Juvenile Justice and Delinquency Prevention, Philadelphia, Hahnemann University.

SPIVACK, G. et N. CIANCI (sous presse), «High Risk Early Behavior Pattern and Later Delinquency», dans J.D. Burchard et S. Burchard (édit.), Prevention of Delinquency and Antisocial Behavior, Washington, DC, U.S. Government Printing Office. 
STOTT, D.H. (1981), «Behavior Disturbance and Failure to Learn : A Study of Cause and Effect», Educational Research, 23, 163-172.

STOTT, D.H. et D.M. WILSON (1968), «The Prediction of Early-Adult Criminality from School-Age Behaviors», International Journal of Social Psychiatry, 14, 5-8.

TANNENBAUM, F. (1938), Crime and Community, New York, Columbia University Press.

TITTLE, C.R., W.J. VILLEMEZ et D.A. SMITH (1978), «The Myth of Social Class and Criminality: An Empirical Assessment of the Empirical Evidence», American Sociological Review, 43, 643-656.

TOBY, J. (1961), «Early Identification and Intensive Treatment of Predelinquents : a Negative View», Social Work, 6, 3-13.

WADSWOTH, M.E.J. (1979), Roots of Delinquency, Infancy, Adolescence and Crime, Oxford, Robertson.

WERNER, E.E. (sous presse), "Vulnerability and Resiliency in Children at Risk for Delinquency : A Longitudinal Study from Birth to Young adulthood», dans J.D. Burchard et S. Burchard (édit.), Prevention of Delinquency and Antisocial Behavior, Washington, D.C., U.S. Government Printing Office.

WEST, D.J. et D.P. Farrington (1973), Who becomes Delinquent?, London, Heinemann.

WOLFGANG, M.E., R.M. FIGLIO et T. SELLIN (1972), Delinquency in a Birth Cohort, Chicago, University of Chicago Press. 